\title{
La bioinformática
}

en la práctica médica: Integración de datos biológicos y clínicos

\author{
Ó scar Coltell $1 \mathrm{a}$, María Arregui ${ }^{1 \mathrm{~b}}$, Antonio Fabregat ${ }^{1 \mathrm{c}}$, \\ O lga Portolés $2 d$.

\section{Integration of clinical and biological data in clinical practice using bioinformatics}

The aim of our work is to describe essential aspects of Medical Informatics, Bioinformatics and Biomedical Informatics, that are used in biomedical research and clinical practice. These disciplines have emerged from the need to find new scientific and technical approaches to manage, store, analyze and report data generated in clinical practice and molecular biology and other medical specialties. It can be also useful to integrate research information generated in different areas of health care. Moreover, these disciplines are interdisciplinary and integrative, two key features not shared by other areas of medical knowledge. Finally, when Bioinformatics and Biomedical Informatics approach to medical investigation and practice are applied, a new discipline, called Clinical Bioinformatics, emerges. The latter requires a specific training program to create a new professional profile. We have not been able to find a specific training program in Clinical Bioinformatics in Spain (Rev Méd Chile 2008; 136: 640-7).

(Key words: Computational Biology; Medical informatics; Public Health Informatics)

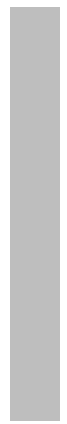
\begin{abstract}
València, Valencia, España.
angeniero Informático y Físico

${ }^{\mathrm{b}} \mathrm{Químico}$

'Ingeniero Informático

dBiólogo
\end{abstract}

Recibido el 25 de mayo, 2007. Aceptado el 17 de julio, 2007.

Financiación: proyectos P11A2005-07 (UJI-BANCAJA, España), GEN2006-26420E_PIA42006-7 (Ministerio de Educación y Ciencia, España), COMBIOMED (RD07/0067/ 0006, ISCIII-FIS, Ministerio de Sanidad, España).

${ }^{1}$ Grupo BioInfoGenómica. Departamento de Lenguajes y Sistemas Informáticos, Universitat Jaume I, Castellón, España. ${ }^{2}$ Grupo EPIGEM. Departamento de Medicina Preventiva y Salud Pública, Ciencia de los Alimentos, Toxicología y Medicina Legal, Universitat de

Correspondencia a: Óscar Coltell. Departamento de Lenguajes y Sistemas Informáticos, Universitat Jaume I. Avda Vicent Sos Baynat, s/n. 12071-Castellón. España. Tel: +34964 728314. Fax:+34 964 728435. E mail: coltell@lsi.uji.es. 
$\mathrm{C}^{\mathrm{o}}$ on la comprobación de que el ADN era la molécula que contenía la información genética, tras el establecimiento en 1953 de la estructura del ADN, y el posterior establecimiento del código genético, quedaron sentadas las bases para el desarrollo de lo que se denomina la Era genómica, caracterizada, tras los avances en las técnicas de manipulación del ADN, por el estudio estructural y funcional de los genes, el análisis comparativo de genomas, la variabilidad genética, así como la estructura de las proteínas. El estudio de la totalidad del genoma humano llevado a cabo por el Proyecto Genoma Humano (PGH) ha dado, pues, lugar a uno de los avances más importantes en la historia de la biología y como se expondrá, a lo largo de este trabajo, de la medicina. Iniciado en 1990, el PGH no tuvo como único objetivo identificar los genes del ADN humano sino también obtener la secuencia de sus bases. Desde el principio, la informática ha sido fundamental en el desarrollo del proyecto, ya que, a finales de los noventa, mediante la automatización y la computación, fue posible secuenciar el genoma humano y debido a estos avances en la tecnología informática así como a la colaboración internacional, el borrador inicial del genoma fue terminado cuatro años antes de lo previsto. También la informática ha sido fundamental para lograr otros objetivos esenciales del PGH como son, guardar la información generada en bases de datos de libre acceso y mejorar las herramientas de análisis y distribución de datos, teniendo en cuenta los aspectos éticos y legales. Pero si bien la primera necesidad fue la de almacenar la información en sistemas de gestión de bases de datos (SGBD) para después recuperarlos y analizarlos, se observa que no sólo el volumen de la información planteaba nuevas necesidades, sino que dicha información necesitaba un tratamiento específico.

Por tanto, los avances que se han producido en el conocimiento del genoma humano han tenido y van a tener en los próximos años un gran impacto tanto en biomedicina, como en las ciencias de la computación, sobre todo en el ámbito de la informática aplicada a la integración de la información genómica en la investigación epidemiológica tradicional ${ }^{1,2}$, ya que a raíz de los nuevos enfoques derivados de la genómica y la postgenómica, resultará difícil concebir la investigación y práctica de la medicina si no es integran- do la información genética, proteómica y otras disciplinas relacionadas, bajo el nombre común de "ómicas", con los datos clínicos, de manera que se espera una considerable repercusión de la aplicación de la informática en la investigación así como su aplicación en el campo de la medicina ${ }^{3}$. Se demanda por tanto, cada día más, programas diseñados específicamente para integrar los resultados de distintas procedencias (secuenciación del ADN, genotipado, microarrays, bases de datos hospitalarias, etc.).

A pesar de lo expuesto anteriormente, la afirmación de Friede acerca de que "La informática en la Salud se ve hoy bajo el mismo prisma que se veía la epidemiología de las enfermedades crónicas y la estadística avanzada hace 20 años: oscura, prohibitivamente compleja, reducida a especialistas, poco práctica, cara y de dudosa utilidad en general"4, sigue siendo verdad más de una década después. La complejidad existe, por la propia materia de estudio y por la necesidad de la participación de profesionales de áreas muy diversas (medicina, biología, estadística, ciencias de la computación, matemáticas, etc.), hasta ahora completamente separadas unas de otras académica y profesionalmente. Sin embargo, la interacción de estas diferentes disciplinas, lejos de reducir la informática a especialistas y convertirla en algo oscuro y poco práctico, o de dudosa utilidad general, ha dado lugar a diferentes disciplinas que, aunque en sus inicios tuvieron un desarmollo independiente, están muy relacionadas entre sí. Fruto de esta interacción, han surgido, la informática médica, bioinformática, la informática biomédica, y la bioinformática clínica. Se comprende que debido a la estrecha correlación que existe entre estas disciplinas, se presten si no a confusión entre los profesionales de la salud y de la informática, sí a la utilización como sinónimos o de forma indistinta de estos términos, quizás debido en parte al debate que aún existe acerca de la naturaleza y objetivos de estas nuevas áreas de conocimiento ${ }^{5}$. No obstante, en la literatura científica estos términos y las correspondientes áreas están bien delimitados.

\section{LA INFORMÁTICA MÉDICA}

En primer lugar, respecto a la informática médica, una primera aproximación, muy simplificada pero intuitiva, sería entenderla como la disciplina que 
aplica la informática a la información clínica. Se trata de un campo bien definido y precursor en el desarrollo e introducción de la informática en el campo de la medicina ${ }^{6}$. La informática médica incluye sistemas automatizados de diagnóstico, de terapia y de comunicación de información de salud. La informática médica, debe responder a la necesidad de gestionar distintos niveles de información sobre salud, datos personales de salud y la historia clínica virtual, fuentes de información médicas de interés, así como bases de datos sobre enfermedades con información para la práctica médica, ensayos clínicos y bases de conocimiento sanitario globales, niveles de información sanitaria que encontrarán su nexo de unión en la historia clínica electrónica (HCE). En el aspecto metodológico, la aproximación de la informática médica es descendente, es decir va desde las manifestaciones clínicas hasta los procesos patológicos, pero sin perder de vista las interacciones que existen entre diversas fuentes de conocimiento que comprenden, por ejemplo, los agentes implicados en la práctica clínica, los recursos provistos por las instituciones y la organización de los agentes en grupos de trabajo y equipos de colaboración, todo ello teniendo en cuenta que el conocimiento está distribuido entre dichos agentes ${ }^{7}$.

En este sistema, en el que están implicados los especialistas en información y los clínicos, el informático es el responsable del diseño, construcción, implantación, gestión y mantenimiento de las soluciones tecnológicas en los sistemas informáticos sanitarios, y resuelve las incidencias técnicas que surgen como consecuencia de la utilización de las soluciones y los sistemas. Mientras que el usuario, el clínico, aplica estas soluciones tecnológicas en su actividad clínica 0 administrativa, pudiendo ser generador de incidencias. Esta disciplina, relativamente genérica y extensa, está enfocada principalmente hacia los aspectos de desarrollo, innovación y producción en los sistemas de información médicos ${ }^{8,9}$.

\section{LA BIOINFORMÁTICA}

La bioinformática, en sentido amplio, se podría definir como la disciplina científica que utiliza la tecnología de la información para organizar, analizar y distribuir información biológica, con la finalidad de responder preguntas complejas en biología ${ }^{10}$, es decir, una disciplina que engloba métodos matemáticos, estadísticos y computacionales para solucionar problemas biológicos usando ADN, ARN, secuencias de aminoácidos e información relacionada ${ }^{11}$.

Las principales aplicaciones de la bioinformática son la gestión, la simulación, la minería de datos y el análisis de la información generada en el PGH, con aplicación también en la predicción de estructuras proteicas, estudios de secuencias y otras actividades derivadas de la investigación en biología. El término simulación hace referencia a la experimentación con un modelo a partir de una hipótesis de trabajo, para comprender la estructura íntima del sistema o realizar una predicción. Por minería de datos se entiende el conjunto de técnicas para la inducción de conocimiento útil a partir de masas ingentes de datos. Debido a que en sus orígenes a través de la bioinformática principalmente se desarrollaban bases de datos genómicas y proteómicas y se construían herramientas software para el análisis y presentación de dichos datos, se la sigue considerando como un campo de apoyo más que como una "ciencia" en sentido real ${ }^{12}$. Quizás, abunde aún más en esa idea de "campo de apoyo", la consideración por parte del National Center for Biotechnology Information (NCBI), de que el fin último de esta disciplina es facilitar el descubrimiento de nuevas ideas biológicas, así como crear perspectivas globales a partir de las cuales se puedan discernir principios unificadores en biología ${ }^{13}$. En el aspecto metodológico, la bioinformática, a diferencia de la informática médica, utiliza una aproximación en sentido ascendente, es decir desde la información genómica a las funciones fisiológicas ${ }^{7}$, teniendo en cuenta a su vez la regulación del fenotipo a través de la interacción gen-ambiente. El desarrollo de esta disciplina es cada vez más importante en el estudio de problemas biomédicos ${ }^{11,14,15}$. Según Kanehisa y Bork, el fin último de la bioinformática es extraer conocimiento a partir de una gran cantidad de datos para obtener una representación de las células y organismos así como predecir sistemas de gran complejidad, como son las redes de interacción en los procesos celulares y el fenotipo de los organismos. En su esquema de representación cronológica acerca del desarrollo de la bioinformática, los autores consi- 
deran que en estos momentos ésta aborda el entendimiento de las funciones del organismo a nivel molecular y celular, mientras que en el futuro esta disciplina investigaría la comprensión de los principios básicos de mayor complejidad de los sistemas biológicos ${ }^{16}$.

\section{LA INFORMÁTICA BIOMÉDICA}

La informática biomédica surge de la faceta investigadora desarrollada por la informática médica (tratamiento de imágenes, diseño de medicamentos, terapias asistidas, etc.), que ha adoptado con rapidez las tecnologías relacionadas con la genómica y la proteómica, con el objetivo de potenciar la investigación clínica y epidemiológica aplicadas ${ }^{17,18}$. La informática biomédica puede verse, pues, como la confluencia entre la informática médica y la bioinformática. Se trata de un nuevo campo interdisciplinario, en la interfase entre ciencias de la computación, las matemáticas, la biología y la medicina ${ }^{19-22}$. De esta forma, la informática biomédica trata aspectos que no están cubiertos por las otras disciplinas, como son la integración de información clínica, genética, medioambiental y el diagnóstico genético, todo ello orientado a encontrar la relación entre problemas clínicos y moleculares en el marco de la salud humana. Si bien la informática médica y la bioinformática convergen en este planteamiento, además de los problemas técnicos y metodológicos, hay que añadir las dificultades formativas de los distintos enfoques disciplinarios ${ }^{23}$. Es más, la complementariedad de conocimientos que hay que integrar no implica sólo un lenguaje común, sino también objetivos de investigación comu$n^{2}{ }^{24}$. En el aspecto metodológico, la informática biomédica utiliza una aproximación transversal entre las disciplinas y vertical entre los distintos niveles de datos que integra.

\section{LA BIOINFORMÁTICA CLÍNICA}

No existen dudas acerca del avance que ha supuesto la bioinformática en el desarrollo de la genética y de las ciencias de la salud, a partir del avance de la tecnología, que ha dado lugar a la posibilidad del estudio de la secuencia y estructu- ra de proteínas, de genes (mutaciones, polimorfismos, comparación de secuencias... ), y al desarrollo de microarrays y de la espectrometría de masas. Estas dos últimas se aplican, respectivamente, para el análisis sistemático de la expresión de genes y la detección de la interacción entre proteínas ${ }^{16}$. Toda esta información, accesible en Internet, ha transformado las bases de datos y su modo de acceso, y debería permitir a los bioinformáticos clínicos aplicarla en la práctica médica, a través de un enfoque nuevo en el tratamiento de enfermedades en lo que se denomina medicina individualizada ${ }^{25}$.

A partir de lo anterior, se entiende que la bioinformática clínica es una aplicación de la bioinformática en distintas áreas y aspectos de la investigación biomédica, así como en la práctica clínica. Así pues, en la mejora de la salud y la prevención de la enfermedad se prevé que el uso de la tecnología de microarrays tenga un gran impacto cuando se puedan resolver los problemas más importantes en el análisis estadístico e interpretación de los resultados y la posterior incorporación rutinaria de estos resultados en la historia clínica. Otras disciplinas implicadas directamente son la proteómica y la farmacología. Con respecto a la proteómica, la bioinformática clínica se plantea el reto de utilizar enormes conjuntos de datos de origen heterogéneo y reforzar la generación de conocimiento, mediante el desarrollo y aplicación de grandes plataformas bioinformáticas que asumen las funciones de sistemas de gestión de datos y bases de datos de conocimiento. En la farmacología, la bioinformática clínica ha servido para identificar dianas terapéuticas, diseñar ensayos clínicos y desarrollar biomarcadores y herramientas toxicogenómicas y farmacogenómicas ${ }^{26}$.

Si bien parece que ésta sea una estrategia a muy largo plazo y alejada todavía de la práctica médica, la bioinformática clínica ha sido y será muy importante en dicha estrategia. Entre otras áreas, se ha aplicado ya en la detección temprana y tratamiento de enfermedades oncológicas, así como en la identificación y reducción de riesgos y prevención de estas enfermedades. Esto ha sido posible gracias a que en la investigación se ha podido integrar la información obtenida a partir de la búsqueda en bases de datos on-line de secuencias genéticas, de proteínas, mutaciones, referencias bibliográficas y redes de regulación génica y rutas metabólicas ${ }^{27}$. 


\section{ASPECTOS FORMATIVOS Y CURRICULARES}

La trayectoria curricular para la formación de especialistas de una nueva disciplina es siempre compleja y está sujeta a revisiones. Este es el caso de la bioinformática clínica, donde se ha de recurrir a otras disciplinas relacionadas, como las que se han mencionado en este trabajo, para poder confeccionar una propuesta curricular. El conocimiento que se ha logrado tras el desarrollo del PGH y las disciplinas generadas a partir del mismo, denominadas genéricamente "ómi-

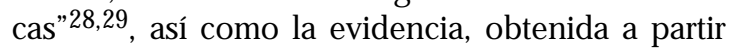
de estudios de epidemiología genómica, de que el estado de salud depende, salvo excepciones, tanto de la interacción de los genes como la de éstos con el ambiente ${ }^{30}$, aún no se pueden incorporar de manera sistemática en la evaluación, prevención y tratamiento de las enfermedades. Aun siendo cierta esta dificultad, resulta cada vez más patente la necesidad de una formación continua de los profesionales de la salud para poder integrar y analizar la compleja información de que se dispone en este momento, dependiente a su vez del progresivo desarrollo tecnológico y científico. Fuera de España, existe la posibilidad de adquirir una formación específica, desde el grado hasta el doctorado, en informática médica $^{31}$, bioinformática ${ }^{32}$ e informática biomédica ${ }^{33}$. En esta sección se analiza brevemente la situación curricular de éstas en España.

La situación en España. En la actualidad, no existe ningún programa universitario de primer o segundo ciclo para la formación en informática médica, bioinformática, informática biomédica o bioinformática clínica. Tampoco se han incorporado en la Reforma de las enseñanzas universitarias ${ }^{34}$ del Ministerio de Educación y Ciencia, en el marco del Espacio Europeo de Educación Superior (EEES) ${ }^{35}$. En el listado de materias básicas por ramas, sólo se contempla la informática en el área de ciencias de la salud. Hay que recordar que, España, como país integrado en el EEES, se encuentra inmerso en un periodo de profundas reformas de la estructura educativa universitaria desde 2003, año de publicación de la primera normativa oficial al respecto ${ }^{36}$.

No obstante, existen algunas iniciativas para la formación en bioinformática como son los cursos de doctorado y másteres, ofertados principalmente en universidades. En el EEES, tanto los programas de doctorado como de máster son la continuación de los grados con una formación mucho más especializada cuyo objetivo es la formación en bioinformática, informática clínica y epidemiología genómica, con el fin de integrarlas y aplicarlas en la práctica clínica. A continuación se relacionan las propuestas actualmente publicadas.

En Cataluña hay varias ofertas. Por una parte, codirigido por la Universidad Pompeu Fabra (UPF) y la Universidad de Barcelona (UB), hay ofertado un título oficial de máster presencial en bioinformática, denominado Máster en Bioinformática para las Ciencias de la Salud (BIOINFO) ${ }^{37}$, que consta de 120 créditos ECTS, se imparte en inglés y es interuniversitario.

Por otra parte, la Universidad Politécnica de Cataluña, ofrece dos másteres oficiales, uno en computación y otro en ingeniería biomédica. El Master in Computing ${ }^{38}$, presencial e impartido en inglés, se realiza durante dos cursos académicos y consta de 120 créditos ECTS. En dicho máster, se contempla el diseño y análisis de algoritmos para resolver problemas, entre otros, de bioinformática y minería de datos. Además, una de las asignaturas de su plan de estudios es precisamente bioinformática. El Master Oficial en Ingeniería Biomédica $^{39}$, semipresencial e impartido conjuntamente por la Universidad de Barcelona y la Universidad Politécnica de Cataluña, pretende la formación de profesionales en actividades relacionadas con los productos y servicios socio-sanitarios, en los aspectos de su diseño, fabricación, evaluación, comercialización, instalación y mantenimiento, así como en la formación sobre la utilización de los equipos médicos y en actividades relacionadas con la preparación para la investigación en un determinado campo de la ingeniería biomédica.

Otras referencias importantes son el máster, el programa de doctorado y el curso de postgrado, propuestos por la Universidad Pontificia de Salamanca Campus de Madrid (UPSAM). El Máster de Gestión de la Información y el Conocimiento en Ciencias de la Salud ${ }^{40}$, presencial y con una carga de 70 créditos ordinarios (equivalentes a 10 horas/ crédito), que incluye un módulo de bioinformática y biotecnología. El Programa de Doctorado en 
Ingeniería Informática. Bioinformática e Informática Biomédica ${ }^{41}$, presencial y semipresencial y con una carga de 32 créditos de doctorado (equivalentes a 10 horas/crédito), ofrece una formación técnica y científica en temas avanzados de informática y comunicaciones, permitiendo conocer nuevos ámbitos de investigación en informática, salud, etc. Finalmente, el Curso Superior de Sistemas de Información, Documentación y Nuevas Tecnologías para la Gestión Clínica y los Sistemas de Salud ${ }^{42}$, está enfocado a la formación para el abordaje de la complejidad del sector de la salud y la gestión de los centros sanitarios. En este curso se analizan las mejoras potenciales que introduciría, en el mencionado sector, el uso de las tecnologías y los sistemas de información, y en particular, la introducción en biotecnología y el estudio de la bioinformática. También se estudia la interacción entre bioinformática e informática médica para facilitar el desarrollo de una medicina personalizada.

Además, hay también una variada oferta de cursos de bioinformática, algunos de ellos como cursos de postgrado, pero que están fuera de los planes de estudios de máster y doctorado. Por ejemplo, la Universidad Internacional de Andalucía oferta varios cursos virtuales: bioinformática estructural y transcriptónica ${ }^{43}$, biología computacional $^{44}$ y complementos de bioinformática ${ }^{45}$.

\section{REFERENCIAS}

1. Cowns FS, Mckusick VA. Implications of the Human Genome Project for medical science. JAMA 2001; 285: 540-4.

2. Knaup P, Ammenwerth E, Brandner R, Brigl B, Fischer G, GaRDE S et al. Towards clinical bioinformatics: advancing genomic medicine with informatics methods and tools. Methods Inf Med 2004; 43: 302-7.

3. Hanai T, Hamada $H$, Окамото M. Application of bioinformatics for DNA microarray data to bioscience, bioengineering and medical fields. J Biosci Bioeng 2006; 101: 377-84.

4. Friede A, Blum HL, Mcdonald M. Public health informatics: how information-age technology can strengthen public health. Annu Rev Public Health 1995; 16: 239-52.

5. Maоjo V, KuLikowski CA. Bioinformatics and Medical Informatics: Collaborations on the Road to
Finalmente, hay que mencionar la labor en la formación en bioinformática llevada a cabo por el área de bioinformática y salud pública del Instituto de Salud Carlos III. Desde su creación, en 1998, viene desarrollando su actividad docente en bioinformática, informática biomédica, microarrays y genómica, para aplicar estas nuevas tecnologías de la información genética en salud pública, práctica clínica, investigación biomédica, etc ${ }^{46}$.

La relación de ofertas mencionadas se asume que no es exhaustiva ya que pueden existir otras, a las que los autores no han tenido acceso, porque se han publicitado en ámbitos restringidos, o se han publicado en boletines de instituciones o empresas sin difusión general. Por otra parte, dada la situación dinámica de algunos de los tipos de ofertas, como son los cursos de postgrado, algunas de las menciones hechas en este artículo pueden variar o desaparecer a posteriori. Y dado que la situación de los másteres oficiales todavía no está consolidada, también puede haber cambios en las ofertas y en las ubicaciones de los portales web después de haberse publicado este artículo. De hecho, desde que los autores empezaron a trabajar en este proyecto, se han sucedido cambios profundos en las ofertas y sus correspondientes ubicaciones en web. Por ello es recomendable ir haciendo revisión de cuando en cuando para tener las direcciones de web siempre actualizadas.

Genomic Medicine? J Am Med Inform Assoc 2003; 10: 515-22.

6. MUSEN MA. Medical informatics: searching for underlying components. Methods Inf Med 2002; 41: 12-9.

7. Hazlenurst B, Gormanb PN, Mcmulena CK. Distributed cognition: An alternative model of cognition for medical informatics. Int J Med Inform 2008; 77: 226-34.

8. Van Bemmel JH, Musen MA (eds.) Handbook of Medical Informatics. Heidelberg, Alemania: Springer-Verlag; 1997.

9. Sackman H. Biomedical Information Technology. Global Social Responsibilities for the Democratic Age. San Diego, CA (USA): Academic Press; 1997.

10. Moldor R, Sturn A, Maurer M, Trajanoski Z. New trends in bioinformatics: from genome sequence to personalized medicine. Exp Gerontol 2003; 38: 1031-6. 
11. Baxevanis AD, Oueuette BF, Boguski M. Bioinformatics: a practical guide to the analysis of genes and proteins. $3^{\text {rd }}$ ed. New York: John Wiley and Sons, Inc., 2004; 1-2.

12. Majo V, Martin F, Crespo J, Biшhardt H. Theory, abstraction and design in medical informatics. Methods Inf Med 2002; 41: 44-50.

13. National Center for Biotechnology Information (NCBI) Home Page. http://www.ncbi.nlm.nih.gov/ . A Science Primer. http://www.ncbi.nlm.nih.gov/ About/primer/bioinformatics.html. [Consultado el 28 de marzo de 2008].

14. Hagen JB. The origins of bioinformatics. Nat Rev Genet 2000; 1: 231-6.

15. GibAS C, JAMBeCK P. Developing bioinformatics computer skills. $1^{\text {st }}$ ed. Sebastopol, CA (USA): O'Reilly; 2001; 3-4. Disponible en http:// books.google.es/books?d.

16. Kanehisa M, Bork P. Bioinformatics in the postsequence era. Nat Genet 2003; 33: 305-10.

17. JoNES RL. «he Internet and Healthcare Information Systems: How Safe Will Patient Data Be?' IS Audit \& Control Journal 1998; I: 25-30.

18. RoBERTs R. "Bioinformatics Analysis of Gene Banks Provides a Treasure Trove for the Functional Genomist". J Mol Cell Cardiol 2000; 32: 1917-19.

19. Cowns FS, Green ED, GutTMacher AE, GuYer MS. A vision for the future of genomics research. Nature 2003; 422: 835-47.

20. Martin-Sánchez F, Iakovidis I, Norager S, Majo V, De Groen P, Van Der Lei J et al. Synergy between medical informatics and bioinformatics: facilitating genomic medicine for future health care. J Biomed Inform 2004; 37: 30-42.

21. MaOjo V, MaRTin-SáncheZ F. Bioinformatics: towards new directions for public health. Methods Inf Med 2004; 43: 208-14.

22. Guttmacher AE, Cowns FS. Welcome to the genomic era. N Engl J Med 2003; 349: 996-8.

23. Martín F, Maojo V. La convergencia entre la bioinformática y la informática médica. I+S 2002; 38: 25-31.

24. TroyansKaya OG. Putting the 'bio' into bioinformatics. Genome Biol 2005; 6: 351.

25. Wulfkuhle JD, Edmiston KH, Lotta LA, Petricoin EF $3^{\text {RD }}$. Technology insight: pharmacoproteomics for cancer-promises of patient-tailored medicine using protein microarrays. Nat Clin Pract Oncol 2006; 3: 256-68.
26. Chang PL. Clinical Bioinformatics. Chang Gung Med J 2005; 28: 2001-11.

27. Kapetanovic IM, Umar A, Khan J. Proceedings: the Applications of Bioinformatics in Cancer Detection. Workshop Ann NY Acad Sci 2004; 1020: 1-9.

28. FISCHER HP. Towards quantitative biology: Integration of biological information to elucidate disease pathways and to guide drug discovery. Biotechnol Annu Rev 2005; 11: 1-68.

29. Corella D, Ordovas JM. Integration of environment and disease into 'omics' analysis. Curr Opin Mol Ther 2005; 7: 569-76.

30. WALACE HM. A model of gene-gene and geneenvironment interactions and its implications for targeting environmental interventions by genotype. Theor Biol Med Model 2006; 9: 3:35.

31. MANDL KD, LeE TH. Integrating medical informatics and health services research: the need for dual training at the clinical health systems and policy levels. J Am Med Inform Assoc 2002; 9: 127-32.

32. HonTS JE. Evolving strategies for the incorporation of bioinformatics within the undergraduate cell biology curriculum. Cell Biol Educ 2003; 2: 233-47.

33. JoHNSON SB. A framework for the biomedical informatics curiculum. AMIA Annu Symp Proc 2003; 331-5. Disponible en http://www.pubmedcentral.nih.gov/ articlerender.fcgi?artid=1480084. [Consultado el $28 \mathrm{de}$ marzo de 2008].

34. Secretaría General del Consejo de Coordinación Universitaria. Reforma de las enseñanzas universitarias. Ministerio de Educación y Ciencia de España, 2008. http://www.mec.es/educa/jsp/ plantilla.jsp?1d=920\&area=ccuniv. [Consultado el 28 de marzo de 2008].

35. Ministerio de Educación y Ciencia. Espacio Europeo de Educación Superior (EEES). Ministerio de Educación y Ciencia de España, 2008. http:// www.mec.es/ universidades/ eees/index.html. [Consultado el 28 de marzo de 2008].

36. Ministerio de Educación y Ciencia. Espacio Europeo de Educación Superior (EEES)- Documentos. Ministerio de Educación y Ciencia de España, 2008. http://www.mec.es/universidades/eees/espana-documentos.html. [Consultado el 28 de marzo de 2008].

37. Universidad Pompeu Fabra. Máster en Bioinformática para las Ciencias de la Salud (BIOINFO). 
http://www.upf.edu/postgrau/es/masters/biomedicina/bioinfo/presenta-cio/index.html. [Consultado el 28 de marzo de 2008].

38. Universitat Politècnica de Catalunya. Master in Computing. http://postgrau.lsi.upc.edu/?cmd=msc_intro. [Consultado el 28 de marzo de 2008].

39. Universitat Politècnica de Catalunya. Máster Oficial en Ingeniería Biomédica. http://www.upc.edu/ enginybiomed $/ \mathrm{htm} / \mathrm{esp} /$ introduccion.htm. [Consultado el 28 de marzo de 2008].

40. Universidad Pontificia de Salamanca. Máster de gestión de la información y el conocimiento en ciencias de la salud. http://www.upsam.com/index.php? Mod=Estudios\&Section=Mostrar\&IdEstudio=76\&Lang=es. [Consultado el 28 de marzo de 2008].

41. Universidad Pontificia de Salamanca. Programa de Doctorado en Ingenieńa Informática. Bioinformática e Informática Biomédica. http://www.upsam.com/ index.php?Mod=Estudios\&Section=Mostrar\&IdEstudio $=114 \& I d O p$ cionMenu=814\&Lang=es. [Consultado el 28 de marzo de 2008].

42. Universidad Pontificia de Salamanca. Curso Superior de Sistemas de Información, Documentación y Nuevas Tecnologías para la Gestión Clínica y los Sistemas de Salud. http://www.upsam.com/ index.php?Mod=Estudios\&Section=Mostrar\&IdEstudio $=1620 \&$ Lang=es. [Consultado el 28 de marzo de 2008].

43. Universidad Internacional de Andalucía. Cursos y jomadas virtuales: Bioinformática estructural y Transcriptónica. http://www.unia.es/index.php?option= com_postunico \&Itemid $=167 \&$ Curso $=$ CF47/ $08 \&$ TipoCurso $=12 \&$ hist $=0$. [Consultado el 28 de marzo de 2008].

44. Universidad Internacional de Andalucía. Cursos y jornadas virtuales: Biología computacional. http:/ / www.unia.es/index.php?option =com_postunico \&Itemid $=167 \quad \&$ Curso $=$ CF48/08\&TipoCurso $=12 \&$ hist $=0$. [Consultado el 28 de marzo de 2008].

45. Universidad Internacional de Andalucía. Cursos y jomadas virtuales: Complementos de Bioinformática. http://www.unia.es/index.php?option= com_postunico \&Itemid $=167 \&$ Curso $=$ CF64 $/ 08 \&$ Tipo Curso $=12 \&$ hist $=0$. [Consultado el 28 de marzo de 2008].

46. Área de Bioinformática y Salud Pública. Actividad Docente. http://biotic.isciii.es/paginas/ActividadDocente.htm. [Consultado el 28 de marzo de 2008]. 